\title{
INDIRECT ABELIAN THEOREMS AND A LINEAR VOLTERRA EQUATION
}

BY

\author{
KENNETH B. HANNSGEN
}

1. Introduction and summary. We study asymptotic behavior of solutions of

$$
x^{\prime}(t)=k-\int_{0}^{t}[a(t-\tau)+c] x(\tau) d \tau, \quad x(0)=x_{0}, \quad\left({ }^{\prime}=\frac{d}{d t}\right)
$$

where $k$ and $x_{0}$ are real, $c \geqq 0$, and $a(t)$ satisfies

(H1) $a(t) \in C(0, \infty) \cap L_{1}(0,1) . a(t)$ is nonnegative and nonincreasing, $\lim _{t \rightarrow \infty} a(t)$ $=0$, and $0<a(0+) \leqq \infty$;

(H2) $a(t)$ is convex downward; i.e., for $0<\varepsilon<1$ and $0<t_{1}<t_{3}<\infty, \varepsilon a\left(t_{1}\right)+$ $(1-\varepsilon) a\left(t_{3}\right) \geqq a\left(t_{2}\right)$, where $t_{2}=\varepsilon t_{1}+(1-\varepsilon) t_{3}$.

By a familiar theorem on Volterra equations, (1.1) has a unique solution in $C^{1}[0, \infty)$. We define $u(t)$ as the solution of (1.1) with $k=0, x_{0}=1$, and we let $w(t)=\int_{0}^{t} u(\tau) d \tau$. It is easily checked that the solution of (1.1) is given by $x_{0} u(t)$ $+k w(t)$.

Treating (1.1) as a special case of a nonlinear equation, Levin proved in [5] that if $a(t) \in C[0, \infty), a(t) \not \equiv a(0)$, and $(-1)^{k} a^{(k)}(t) \geqq 0$ for $0<t<\infty, k=0,1,2,3$, then

$$
\lim _{t \rightarrow \infty} u(t)=0
$$

and

(1.3).(i) If $c+a(t) \in L_{1}(0, \infty)$ (in particular, $c=0$ ), then

$$
\lim _{t \rightarrow \infty} w(t)=\left(\int_{0}^{\infty} a(t) d t\right)^{-1}
$$

(ii) If $c>0$, then $\lim _{t \rightarrow \infty} w(t)=0$.

Levin also conjectured that

(1.4) If $c=0, a(t) \notin L_{1}(0, \infty)$, then $\lim _{t \rightarrow \infty} w(t)=0$.

The present theorem shows that (H1) and (H2) together are nearly sufficient for (1.2), (1.3), and (1.4); in particular, Levin's conjecture is proved. The theorem also exhibits a class of kernels satisfying $(\mathrm{H} 1)$ and $(\mathrm{H} 2)$ but for which a different asymptotic behavior from (1.2), (1.3), and (1.4) can be established.

Received by the editors September 13, 1968 and, in revised form, February 3, 1969. 
More specifically, note that if $a(t)$ is given by

$$
\begin{aligned}
& \text { (a) } \quad a(t)=\sum_{k=1}^{\infty} \delta_{k}\left(1-\frac{\min \left\{t, k t_{0}\right\}}{k t_{0}}\right), \quad t_{0}=\frac{2 \pi}{\tau_{0}}>0 \\
& \text { (b) } \delta_{k} \geqq 0, \quad 0<\delta \equiv \sum_{k=1}^{\infty} \delta_{k}=a(0)<\infty \\
& \text { (c) } \Omega \equiv\left\{k \mid \delta_{k}>0\right\} \text { has no common divisor }>1,
\end{aligned}
$$

then $a(t)=\sum_{j=k}^{\infty} \delta_{j}-t \sum_{j=k}^{\infty}\left(\delta_{j} / j t_{0}\right),(k-1) t_{0} \leqq t \leqq k t_{0}$. It follows that $a(t)$ is continuous, and on each interval $(k-1) t_{0} \leqq t \leqq k t_{0}$ it is linear with slope $-\sum_{k=j}^{\infty}\left(\delta_{j} / j t_{0}\right)$. Then $a(t)$ satisfies (H1) and (H2). When (1.5) holds, we may also have

$$
\omega \equiv \sqrt{ }(\delta+c)=j \tau_{0}, \quad j=\text { positive integer. }
$$

We will establish (1.2), (1.3), and (1.4) when $a(t)$ satisfies (H1), (H2), and

(H3) a(t) admits no representation (1.5) such that (1.6) holds.

Complementary to (H3) is

(H4) $a(t)$ satisfies (1.5) and (1.6).

When (H4) holds, we define $\gamma=(3 \delta+2 c) /(\delta+c)$ and let

$$
u_{1}(t)=u(t)-2 \gamma^{-1} \cos \omega t
$$

and

$$
w_{1}(t)=w(t)-2(\gamma \omega)^{-1} \sin \omega t
$$

We prove

THEOREM. Let $c \geqq 0$, and let $a(t)$ satisfy $(\mathrm{H} 1)$ and $(\mathrm{H} 2)$. Then

(i)

$$
\begin{aligned}
& \lim _{t \rightarrow \infty} u(t)=0, \quad \text { if }(\mathrm{H} 3) \text { holds, } \\
& \lim _{t \rightarrow \infty} u_{1}(t)=0, \text { if (H4) holds. }
\end{aligned}
$$

(ii) If $c+a(t) \notin L_{1}(0, \infty)$,

$$
\begin{aligned}
& \lim _{t \rightarrow \infty} w(t)=0, \quad \text { if }(\mathrm{H} 3) \text { holds, } \\
& \lim _{t \rightarrow \infty} w_{1}(t)=0 \text {, if (H4) holds. }
\end{aligned}
$$

(iii) If $c+a(t) \in L_{1}(0, \infty)$,

$$
\begin{aligned}
& \lim _{t \rightarrow \infty} w(t)=\left(\int_{0}^{\infty} a(t) d t\right)^{-1}, \text { if }(\mathrm{H} 3) \text { holds }, \\
& \lim _{t \rightarrow \infty} w_{1}(t)=\left(\int_{0}^{\infty} a(t) d t\right)^{-1}, \text { if }(\mathrm{H} 4) \text { holds. }
\end{aligned}
$$

Generalizations of the results in [5] to nonlinear versions of (1.1) are given by Levin and Nohel in [7]. A result of Friedman (Theorem $C$ of [3]) implies (1.2) and (1.4) for $c+a(t)=t^{-\alpha}, 0<\alpha<1$. Halanay [4] studied a nonlinear equation 
including (1.1) with $k=0$ when $c+a(s-\tau)-\varepsilon_{0} e^{-\alpha|s-\tau|}$ is a positive kernel on $\{0 \leqq s \leqq t, 0 \leqq \tau \leqq t\}$ for all $t \geqq 0$ and some $\varepsilon_{0}>0, \alpha>0$.

The Laplace transform argument of our proof resembles the proofs of the "indirect abelian" theorems in [2, pp. 265-275]. Such theorems were used by Levin and Nohel in [6] to find an asymptotic expansion as $t \rightarrow \infty$ of solutions of an equation similar to (1.1) but where the kernel is, among other things, completely monotonic on $[0, \infty)$.

Throughout the discussion $S$ denotes the subset of the complex plane given by

$$
S=\{s \mid \operatorname{Re} s \geqq 0, s \neq 0\} \text {. }
$$

We define

$$
A(s)=\lim _{T \rightarrow \infty} \int_{0}^{T} e^{-s t} a(t) d t, \quad s \in S .
$$

Then $A(s)$ is the Laplace transform of $a(t)$; similarly let $X(s)$ be the Laplace transform of $X(t)$. Taking Laplace transforms formally in (1.1), we obtain $X(s) p(s)$ $=x_{0}+(k / s)$, where $p(s)=(c / s)+A(s)+s$. In Lemma 5 we show that when (H3) holds, $p(s) \neq 0$ for $s \in S$. Then

$$
X(s)=\left(x_{0}+(k / s)\right) / p(s), \quad s \in S .
$$

The complex inversion formula for Laplace transforms, together with contour integration and some estimates on $A(s)$, yields

$$
x(t)=\lim _{\varepsilon \rightarrow 0} \frac{1}{2 \pi}\left[\int_{-\infty}^{-\varepsilon}+\int_{\varepsilon}^{\infty}\right] e^{i \tau t} X(i \tau) d \tau, \quad t>0,
$$

where for each $\varepsilon>0$ the integrals are uniformly convergent in $t \geqq T>0$. The Riemann-Lebesgue theorem and other abelian arguments then yield our results.

When (H4) holds, we find that $p(s)$ has exactly the two zeros $s= \pm i \omega$ in $S$. A formula similar to (1.7) but with principal values taken also at $\tau= \pm \omega$ is used in this case.

$\$ 2$ presents a sequence of lemmas concerning $a(t)$ and $A(s)$. The theorem is proved in $\$ 3$.

Much of the research presented here appeared in the author's Ph.D. thesis (University of Wisconsin, 1968). The author wishes to thank his thesis director, Professor J. J. Levin, for many suggestions used here. Most of this research was done while the author held an NSF Graduate Fellowship.

\section{The Laplace transform of $a(t)$.}

LEMMA 1. Let $\sigma>0$, and let $a(t)$ satisfy $(\mathrm{H} 1)$. Then

(i) $e^{-\sigma t} a(t)$ satisfies $(\mathrm{H} 1)$.

(ii) If $a(t)$ satisfies $(\mathrm{H} 2)$, so does $e^{-\sigma t} a(t)$.

Proof. (i) Obvious. 
(ii) Since $a(t)$ is continuous, it suffices to prove the convexity relation in (H2) with $\varepsilon=1 / 2$. Set $a_{i}=a\left(t_{i}\right)$ and $b_{i}=\exp \left(-\sigma t_{i}\right), i=1,2,3$. Then $a_{i}, b_{i} \geqq 0, a_{1}-a_{2} \geqq a_{2}$ $-a_{3} \geqq 0, b_{1}-b_{2}>b_{2}-b_{3}>0$. Hence $a_{1} b_{1}-a_{2} b_{2}=a_{1}\left(b_{1}-b_{2}\right)+b_{2}\left(a_{1}-a_{2}\right) \geqq a_{2}\left(b_{2}-b_{3}\right)$ $+b_{3}\left(a_{2}-a_{3}\right)=a_{2} b_{2}-a_{3} b_{3}$, and (ii) is proved.

We state without proof the following easy consequence of convexity:

LEMMA 2. If $a(t)$ satisfies (H2), then for any $\delta>0$, the function $a(t)-a(t+\delta)$ is nonincreasing.

Lemma 3. Let a(t) satisfy (H1). Then

(i) $A(s)$ is defined, finite, and continuous in $S . A(s)$ is holomorphic in $\{\operatorname{Re} s>0\}$.

(ii) For $\sigma+i \tau \in S, \tau \neq 0$,

$$
\begin{aligned}
|\operatorname{Im} A(\sigma+i \tau)| & \leqq \int_{0}^{\pi /|\tau|} a(t) \sin |\tau| t d t \\
& \leqq \int_{0}^{\pi /|\tau|} a(t) d t
\end{aligned}
$$

and

$$
|\operatorname{Re} A(\sigma+i \tau)| \leqq \int_{0}^{\pi / 2|\tau|} a(t) d t,
$$

so that $|A(\sigma+i \tau)| \rightarrow 0$ as $|\tau| \rightarrow \infty$, uniformly in $0 \leqq \sigma<\infty$.

(iii) If $a(t)$ also satisfies $(\mathrm{H} 2)$ and $\sigma+i \tau \in S$, then

$$
\begin{aligned}
|A(\sigma+i \tau)| & \geqq \frac{1}{\sqrt{ } 2} \int_{0}^{\pi / 2|\tau|} \cos \tau t e^{-\sigma t} a(t) d t \\
& \geqq \frac{1}{2 \sqrt{ } 2} \int_{0}^{\pi / 3|\tau|} e^{-\sigma t} a(t) d t
\end{aligned}
$$

(the case $\tau=0, \tau^{-1}=\infty$, is included); and if $\tau>0$,

$$
\lim _{T \rightarrow \infty} \int_{0}^{T} a(t) \sin \tau t d t+\lim _{T \rightarrow \infty} \int_{\pi / 2 \tau}^{T} a(t) \cos \tau t d t \geqq 0 .
$$

Proof. (i) For $s=\sigma+i \tau \in S, \tau>0, T>0$, define

$$
\begin{aligned}
& \phi(T, s)=\int_{0}^{T} a(t) e^{-\sigma t} \cos \tau t d t \\
& \psi(T, s)=\int_{0}^{T} a(t) e^{-\sigma t} \sin \tau t d t .
\end{aligned}
$$

Since $a(t) \rightarrow 0$ as $t \rightarrow \infty, A(s)$ has a nonpositive abscissa of convergence (see e.g. [8, Chapter II]) so that $A(s)$ is holomorphic in $\{\operatorname{Re} s>0\}$ with

$$
[A(s)]^{*}=A\left(s^{*}\right)=\lim _{T \rightarrow \infty}[\phi(T, s)+i \psi(T, s)]
$$

for $\operatorname{Im} s>0, *=$ complex conjugate. 
For any $T>0, \phi$ and $\psi$ are continuous functions of $s$. (H1) and Lemma 1(i) show that $e^{-\sigma t} a(t)$ is nonnegative and nonincreasing on $(0, \infty)$. For $s=\sigma+i \tau, \tau>0$, $T_{1}, T_{2} \geqq\left(n+\frac{1}{2}\right) \pi / \tau, n=$ nonnegative integer,

$$
\left|\phi\left(T_{1}, s\right)-\phi\left(T_{2}, s\right)\right| \leqq \int_{(n+1 / 2) \pi / \tau}^{(n+3 / 2) \pi / \tau} a(t) d t
$$

and similarly for $\psi$. Since $a(t) \rightarrow 0$ as $t \rightarrow \infty, \phi(T, s)$ and $\psi(T, s)$ converge as $T \rightarrow \infty$, uniformly in any set of the form

$$
S \cap\left\{\sigma+i \tau \mid 0<\tau_{0} \leqq \tau \leqq \tau_{1}<\infty\right\}
$$

to continuous functions $\phi(s)$ and $\psi(s)$. Comparing this with (2.5), we see that (i) is proved.

(ii) Since $\operatorname{Re} A(\sigma+i \tau)$ and $\operatorname{Im} A(\sigma+i \tau)$ are respectively even and odd in $\tau$, we may assume $\tau>0$. Since for each $T>0$ we have

$$
\begin{aligned}
|\phi(T, \sigma+i \tau)| & \leqq \int_{0}^{\pi / 2 \tau} a(t) e^{-\sigma t} \cos \tau t d t \\
& \leqq \int_{0}^{\pi / 2 \tau} a(t) d t
\end{aligned}
$$

(2.2) holds. (2.1) is obtained similarly.

(iii) The case $\tau=0$ is trivial, and by symmetry of $\operatorname{Re} A(\sigma+i \tau)$ and $\operatorname{Im} A(\sigma+i \tau)$ in $\tau$, we may assume $\tau>0$. Note first that since $A(i \tau)=\phi(i \tau)-i \psi(i \tau)$,

$$
\sqrt{ } 2|A(i \tau)| \geqq|\phi(i \tau)|+|\psi(i \tau)| \geqq \phi(i \tau)+\psi(i \tau) .
$$

But

$$
\phi(i \tau)=\int_{0}^{\pi / 2 \tau} a(t) \cos \tau t d t+\lim _{n \rightarrow \infty} \sum_{k=1}^{n} \int_{2(k-1) \pi / \tau}^{2 k \pi / \tau} a\left(t+\frac{\pi}{2 \tau}\right) \cos \left[\tau\left(t+\frac{\pi}{2 \tau}\right)\right] d t
$$

and

so (2.6) becomes

$$
\psi(i \tau)=\lim _{n \rightarrow \infty} \sum_{k=1}^{n} \int_{2(k-1) \pi / \tau}^{2 k \pi / \tau} a(t) \sin \tau t d t
$$

$$
\begin{aligned}
|\sqrt{ } 2 A(i \tau)| & -\int_{0}^{\pi / 2 \tau} a(t) \cos \tau t d t \\
& \geqq \lim _{n \rightarrow \infty} \sum_{k=1}^{n} \int_{2(k-1) \pi / \tau}^{2 k \pi / \tau}\left\{a(t) \sin \tau t+a\left(t+\frac{\pi}{2 \tau}\right) \cos \left[\tau\left(t+\frac{\pi}{2 \tau}\right)\right]\right\} d t .
\end{aligned}
$$

We note that the right-hand side of (2.7) equals the left-hand side of (2.4) and that both (2.3) for $\sigma=0$ and (2.4) will follow if we show that this right-hand side is nonnegative. But for any integer $k \geqq 1$,

$$
\begin{aligned}
\int_{2(k-1) \pi / \tau}^{2 k \pi / \tau}\left\{a(t) \sin \tau t+a\left(t+\frac{\pi}{2 \tau}\right) \cos \left[\tau\left(t+\frac{\pi}{2 \tau}\right)\right]\right\} d t \\
=\int_{0}^{\pi / \tau} \sin \tau t\left[a\left(t+x_{0}\right)-a\left(t+x_{1}\right)-a\left(t+x_{2}\right)+a\left(t+x_{3}\right)\right] d t
\end{aligned}
$$


where $x_{j}=2(k-1) \pi / \tau+j \pi / 2 \tau, j=0,1,2,3$, and Lemma 2 with $\delta=\pi / 2 \tau$ shows that the integrand is nonnegative. For (2.3) with $\sigma>0$, we apply (2.3) with $\sigma=0$ to the function $b(t)=e^{-\sigma t} a(t)$, which satisfies (H1) and (H2) by Lemma 1 , and which has Laplace transform $B(s)=A(s+\sigma)$. This completes the proof of Lemma 3.

CoRollary 3.1. Let a(t) satisfy (H1). Then $|s A(s)| \rightarrow 0$ as $s \rightarrow 0, s \in S$.

Proof. We let $s=\sigma+i \tau$. Applying (2.1) and (2.2) to the function $b(t)=e^{-\sigma t} a(t)$, we have

$$
|s A(s)|=|s||B(i \tau)| \leqq \sqrt{ } 2|s| \int_{0}^{\pi /|\tau|} e^{-\sigma t} a(t) d t,
$$

when $\tau \neq 0$ and, trivially, also when $\tau=0, \sigma>0$. Thus if $\sigma \geqq|\tau|,|s A(s)| \leqq 2 \sigma \int_{0}^{\infty} e^{-\sigma t}$ $\cdot a(t) d t=2 \sigma A(\sigma)$, while if $|\tau|>\sigma$, we have $|s A(s)| \leqq 2|\tau| \int_{0}^{\pi /|\tau|} a(t) d t$; one of these estimates is valid for each $s \in S$. But since $a(t) \rightarrow 0$ as $t \rightarrow \infty$,

$$
\int_{0}^{x} a(t) d t=o(x) \quad(x \rightarrow \infty) .
$$

It follows that $|\tau| \int_{0}^{\pi /|\tau|} a(t) d t \rightarrow 0$ as $\tau \rightarrow 0$; and (2.8) together with an elementary abelian theorem for Laplace transforms [8, p. 182] gives $\sigma A(\sigma) \rightarrow 0$ as $\sigma \rightarrow 0+$. In view of our estimates for $|s A(s)|$, the corollary is proved.

Corollary 3.2. Let $a(t)$ satisfy $(\mathrm{H} 1)$ and $(\mathrm{H} 2)$, and suppose that $a(t) \notin L_{1}(0, \infty)$. Then $[s+A(s)]^{-1} \rightarrow 0$ as $s \rightarrow 0, s \in S$.

Proof. By Lemma 3(iii),

$$
\begin{aligned}
|A(s)|=|A(\sigma+i \tau)| & \geqq \frac{1}{2 \sqrt{ } 2} \int_{0}^{\pi / 3|\tau|} e^{-\sigma t} a(t) d t \\
& \geqq m \int_{0}^{\pi / 3|s|} a(t) d t,
\end{aligned}
$$

where $m^{-1}=2 \sqrt{ } 2 e^{\pi / 3}$. Then for sufficiently small $|s|$,

$$
\left|[s+A(s)]^{-1}\right| \leqq 2\left(m \int_{0}^{\pi / 3|s|} a(t) d t\right)^{-1}=o(1) \quad(|s| \rightarrow 0) .
$$

LEMMA 4. Suppose a(t) satisfies $(\mathrm{H} 1)$ and $(\mathrm{H} 2)$. Then exactly one of the following two cases holds:

I. Either (i) $a(0+)=\infty$ or (ii) $a(0) \equiv a(0+)<\infty$ and $\forall \tau>0$ there exists an integer $k=k(\tau)>0$ such that

$$
a\left(\frac{2(k-1) \pi}{\tau}\right)-2 a\left(\frac{2(k-1) \pi+\pi}{\tau}\right)+a\left(\frac{2 k \pi}{\tau}\right)>0 .
$$

II. (i) There exists a positive number $\tau_{0}$ and a sequence $\left\{\delta_{k}\right\}_{k=1}^{\infty}$ such that (1.5) holds. 
(ii) The numbers $\tau_{0}, t_{0}$, and $\delta$ and the sequence $\left\{\delta_{k}\right\}$ are determined uniquely by (1.5). All positive $\tau$ such that

$$
a\left(\frac{2(k-1) \pi}{\tau}\right)-2 a\left(\frac{2(k-1) \pi+\pi}{\tau}\right)+a\left(\frac{2 k \pi}{\tau}\right)=0, \quad k=1,2, \ldots
$$

are integral multiples of $\tau_{0}$.

(iii) The Laplace transform of $a(t)$ is given by

$$
A(s)=\frac{\delta}{s}+\frac{1}{s^{2}} \sum_{k=1}^{\infty} \frac{\delta_{k}}{k t_{0}}\left(\exp \left[-s k t_{0}\right]-1\right), \quad s \in S .
$$

Proof. First we note that a representation (1.5) implies (2.10) with $\tau=\tau_{0}$, so cases I and II exclude each other.

Now suppose we are not in case I, so that (2.10) holds for some $\tau=\tau_{1}$. Let $J$ denote the set of positive integers $j$ such that (2.10) holds when $\tau=\tau_{1} / j$. Then $1 \in J$. Also, $J$ is a finite set; for $(\mathrm{H} 1),(\mathrm{H} 2)$, and (2.10) with $k=1, \tau=\tau_{1} / j, j \in J$, show that $a(t)$ is linear with negative slope on $\left[0,2 j \pi / \tau_{1}\right]$ whenever $j \in J$. We let $j_{0}$ be the largest $j \in J$, and set $\tau_{0}=\tau_{1} / j_{0}$. Then for any integer $j>1$, (2.10) does not hold with $\tau=\tau_{0} / j$.

By convexity, (2.10) with $\tau=\tau_{0}$ shows that $a(t)$ is linear on each interval $2(k-1) \pi / \tau_{0} \leqq t \leqq 2 k \pi / \tau_{0}$; we let $-\lambda_{k}$ be its slope there. By (H1) and (H2),

$$
\lambda_{1} \geqq \lambda_{2} \geqq \lambda_{3} \geqq \cdots \geqq 0, \text { and } \lim _{k \rightarrow \infty} \lambda_{k}=0 \text {. }
$$

We define $t_{0}=2 \pi / \tau_{0}$ and $\delta_{k}=k t_{0}\left(\lambda_{k}-\lambda_{k+1}\right) \geqq 0, k=1,2,3, \ldots$ Then on the interval $(k-1) t_{0} \leqq t \leqq k t_{0}$, the function defined by the right-hand side of (1.5a) has the value

$$
\begin{aligned}
\sum_{j=k}^{\infty} \delta_{j}\left(1-\frac{t}{j t_{0}}\right) & =\sum_{j=k}^{\infty}\left(\lambda_{j}-\lambda_{j+1}\right)\left(j t_{0}-t\right) \\
& =\lambda_{k}\left(k t_{0}-t\right)+\sum_{j=k+1}^{\infty} \lambda_{j} t_{0} \\
& =\int_{\infty}^{t} d a(\tau)=a(t) .
\end{aligned}
$$

This proves (1.5a) and (1.5b).

For (1.5c), we note that if $j>1$ divides all $k$ in $\Omega$, then

$$
a(t)=\sum_{k=1}^{\infty} \delta_{j k}\left(1-\frac{\min \left\{t, j k t_{0}\right\}}{j k t_{0}}\right),
$$

and as in the proof of (1.5a), $a(t)$ is linear on $2 j(k-1) \pi / \tau_{0} \leqq t \leqq 2 j k \pi / \tau_{0}, k=1,2, \ldots$. But then (2.10) holds with $\tau=\tau_{0} / j$, and we chose $\tau_{0}$ so as to make this impossible. This completes the proof that II(i) holds when I does not hold.

To prove (ii), suppose

$$
a(t)=\sum_{k=1}^{\infty} \delta_{k}^{\prime}\left(1-\frac{\min \left\{t, k t_{0}^{\prime}\right\}}{k t_{0}^{\prime}}\right), \quad t_{0}^{\prime}=2 \pi / \tau_{0}^{\prime}
$$


with corresponding $\left(1.5 \mathrm{~b}^{\prime}\right),\left(1.5 \mathrm{c}^{\prime}\right)$. Let $k_{1}<k_{2}<k_{3}<\cdots$ be all the elements of $\Omega$, and $k_{1}^{\prime}<k_{2}^{\prime}<\cdots$ all the elements of $\Omega^{\prime}$. By $\left(1.5 \mathrm{a}, \mathrm{a}^{\prime}\right)$, for each $i$

(2.12) $k_{i} t_{0}=k_{i}^{\prime} t_{0}^{\prime}=\max \{x \mid$ slope of $a(t)$ has exactly $i$ different values on $(0, x)\}$.

In particular, $t_{0} / t_{0}^{\prime}=k_{1}^{\prime} / k_{1}=$ rational number, so $t_{0} / t_{0}^{\prime}=p / q$, where $p$ and $q$ are relatively prime positive integers. Then for each $i$, by (2.12), $k_{i} p / q=k_{i}^{\prime}=$ integer. By (1.5c), $q=1$, so by $\left(1.5 \mathrm{c}^{\prime}\right)$ also $p=1$ and $t_{0}=t_{0}^{\prime}$. By $\left(1.5 \mathrm{a}, \mathrm{a}^{\prime}\right), \tau_{0}=\tau_{0}^{\prime}$. By $\left(1.5 \mathrm{a}^{\prime}\right)$, the slope $-\lambda_{k}$ of $a(t)$ in $\left[(k-1) t_{0}, k t_{0}\right]$ is $-\sum_{j=k}^{\infty}\left(\delta_{j}^{\prime} \mid j t_{0}\right)$, so by the definition of $\delta_{k}$ in the proof of (i), $\delta_{k}^{\prime}=\left(\lambda_{k}-\lambda_{k+1}\right) k t_{0}=\delta_{k}$. (1.5b, $\left.\mathrm{b}^{\prime}\right)$ give $\delta=\delta^{\prime}$, and uniqueness is proved.

Any $\tau$ satisfying (2.10) leads, as in (i), to a representation $\left(1.5^{\prime}\right)$ with $\tau_{0}^{\prime}=\tau / j$. By uniqueness $j \tau_{0}=j \tau_{0}^{\prime}=\tau$, and (ii) is proved.

(iii) This follows from (1.5) by direct computation. This completes the proof of Lemma 4.

Lemma 5. Let $a(t)$ satisfy $(\mathrm{H} 1)$ and $(\mathrm{H} 2)$, and let $c \geqq 0$. Define

$$
p(s)=(c / s)+A(s)+s, \quad s \in S .
$$

Then

(i) $p(s)$ has no zeros in $S$ if $(\mathrm{H} 3)$ holds. If $(\mathrm{H} 4)$ holds $p(s)$ has exactly the two zeros $s= \pm i \omega$ in $S$.

(ii) When (H4) holds,

$$
|p(s)-\gamma(s-i \omega)|=o(|s-i \omega|) \quad(s \rightarrow i \omega, s \in S),
$$

where $\gamma=(3 \delta+2 c) /(\delta+c)$.

Proof. (i) First, (2.11) shows that $p( \pm i \omega)=0$ when (H4) holds.

For $s=i \tau \neq 0$,

$$
\operatorname{Re} A(i \tau)=\lim _{n \rightarrow \infty} \sum_{k=1}^{n} \int_{0}^{2 \pi /|\tau|} a\left(\frac{2(k-1) \pi}{|\tau|}+t\right) \cos \tau t d t .
$$

But for each $k$, the integral in the sum is equal to

$$
\int_{0}^{\pi / 2|\tau|}\left[a\left(x_{0}+t\right)-a\left(x_{1}-t\right)-a\left(x_{1}+t\right)+a\left(x_{2}-t\right)\right] \cos \tau t d t,
$$

where $x_{j}=[2(k-1)+j] \pi /|\tau|, j=0,1,2$. Lemma 2 with $\delta=(\pi /|\tau|)-2 t$ shows that the integrand is nonnegative. Furthermore, Lemma 4 shows that if $a(t)$ is in case I of Lemma 4 or in case II with $j \tau_{0} \neq|\tau|, j=1,2,3, \ldots$, then there exists $k$ such that the integrand is positive at $t=0$, and by continuity on an interval $0 \leqq t<\varepsilon$; for this $k$ the integral is positive. We conclude that $\operatorname{Re} A(i \tau) \geqq 0$, and if $\operatorname{Re} A(i \tau)=0$, then $a(t)$ is in case II of Lemma 4 with $\tau=j \tau_{0}, j=$ integer.

By Lemma 1, if $\sigma>0$, the function $e^{-\sigma t} a(t)$ satisfies (H1) and (H2). Thus for $\sigma>0$ (by the preceding paragraph for $\tau \neq 0$ and trivially for $\tau=0), \operatorname{Re} A(\sigma+i \tau)$ $=\int_{0}^{\infty}\left[e^{-\sigma t} a(t)\right] \cos \tau t d t \geqq 0$. 
To apply these remarks, we suppose that $p(s)=0, s=\sigma+i \tau \in S$. Then

$$
0=\operatorname{Re} p(s)=c \sigma /\left(\sigma^{2}+\tau^{2}\right)+\operatorname{Re} A(\sigma+i \tau)+\sigma .
$$

Therefore $\sigma=0$ and $a(t)$ is in case II with $\tau=j \tau_{0}, j=$ integer. But then, using (2.11),

$$
0=\operatorname{Im} p(s)=-(c+\delta) / j \tau_{0}+j \tau_{0} ;
$$

i.e., $c+\delta=\left(j \tau_{0}\right)^{2}=\tau^{2}$, so that (H4) holds and $s= \pm i \omega$. This proves (i).

(ii) By (2.11),

$$
p(s)-s+\frac{\omega^{2}}{s}+\frac{\delta}{s^{2}}(s-i \omega)=\left[\frac{1}{s^{2}} \sum_{k=1}^{\infty} \frac{\delta_{k}}{k t_{0}}\left(\exp \left[-s k t_{0}\right]-1\right)\right]-\frac{\delta}{s^{2}}(s-i \omega) .
$$

On the left-hand side we expand $s, \omega^{2} / s$, and $\delta / s^{2}$ in Taylor series about $i \omega$; on the right we rearrange terms. Then

$$
\begin{array}{r}
p(s)-\gamma(s-i \omega)+O\left(|s-i \omega|^{2}\right)=s^{-2} \sum_{k=1}^{\infty} \frac{\delta_{k}}{k t_{0}}\left(\exp \left[-s k t_{0}\right]-1+s k t_{0}-i \omega k t_{0}\right) \\
(s \rightarrow i \omega, s \in S) .
\end{array}
$$

For $\operatorname{Re} s \geqq 0$,

$$
\begin{aligned}
\left|\left(\exp \left[-s k t_{0}\right]-1+s k t_{0}-i \omega k t_{0}\right) / k t_{0}\right| & \leqq\left|\exp \left[-s k t_{0}\right]-1\right|\left|k t_{0}+\right| s-i \omega \mid \\
& \leqq 2\left|k t_{0}+\right| s-i \omega \mid .
\end{aligned}
$$

On the other hand, the power series expansion of $\exp \left(-s k t_{0}\right)$ about $s=i \omega$ yields for $k|s-i \omega| \leqq 1$

$$
\begin{aligned}
\left|\left(\exp \left[-s k t_{0}\right]-1+s k t_{0}-i \omega k t_{0}\right) / k t_{0}\right| & =\left|\sum_{j=2}^{\infty}\left[(s-i \omega)\left(-k t_{0}\right)\right]^{j} / j !\right| \mid k t_{0} \\
& =k|s-i \omega|^{2}\left|\sum_{j=0}^{\infty} t_{0}^{j+2} /(j+2) !\right| \mid t_{0} \\
& \leqq\left(\exp \left[t_{0}\right] k / t_{0}\right)|s-i \omega|^{2} .
\end{aligned}
$$

Using these two estimates, we have for $\operatorname{Re} s \geqq 0$

$$
\begin{aligned}
& \left|\sum_{k=1}^{\infty} \frac{\delta_{k}}{k t_{0}}\left(\exp \left[-s k t_{0}\right]-1+s k t_{0}-i \omega k t_{0}\right)\right| \\
& \quad \leqq \frac{\exp \left[t_{0}\right]}{t_{0}}|s-i \omega|^{2} \sum_{k=1}^{n(s)} k \delta_{k}+|s-i \omega| \sum_{k=n(s)+1}^{\infty} \delta_{k}+\frac{2}{t_{0}} \sum_{k=n(s)+1}^{\infty} \delta_{k} / k,
\end{aligned}
$$

where $n(s)$ is the greatest integer such that $n(s)|s-i \omega| \leqq 1$.

By $(1.5 \mathrm{a}), a(t) \geqq \sum_{k=1}^{m} \delta_{k}\left[1-\left(\min \left\{t, k t_{0}\right\}\right) / k t_{0}\right]$ for $m=1,2,3, \ldots$ Since

$$
\int_{0}^{k t_{0}} \delta_{k}\left(1-t / k t_{0}\right) d t=k t_{0} \delta_{k} / 2
$$

we have $2 \int_{0}^{m t_{0}} a(t) d t \geqq t_{0} \sum_{k=1}^{m} k \delta_{k}$. Hence

$$
|s-i \omega| \sum_{k=1}^{n(s)} k \delta_{k} \leqq \frac{2}{t_{0} n(s)} \int_{0}^{t_{0} n(s)} a(t) d t \rightarrow 0 \quad \text { as }|s-i \omega| \rightarrow 0,
$$


since $a(t) \rightarrow 0$. Also, since $\sum \delta_{k}<\infty$,

$\sum_{k=n(s)+1}^{\infty} \delta_{k}+\frac{2}{t_{0}|s-i \omega|} \sum_{k=n(s)+1}^{\infty} \delta_{k} \mid k \leqq\left(1+2 / t_{0}\right) \sum_{k=n(s)+1}^{\infty} \delta_{k} \rightarrow 0 \quad$ as $|s-i \omega| \rightarrow 0$.

Combining this with (2.14), (2.15), and (2.16), we have (2.13), and Lemma 5 is proved.

3. Proof of theorem. Integrating (1.1), we obtain

$$
x(t)=x_{0}+k t-\int_{0}^{t} f(t-\tau) x(\tau) d \tau,
$$

where $0 \leqq f(t) \equiv \int_{0}^{t}[a(\tau)+c] d \tau \leqq \int_{0}^{1} a(\tau) d \tau+c+[a(1)+c] t$. By a standard result on Volterra equations $[1, \S 7.6], x(t)$ satisfies an inequality

$$
|x(t)| \leqq B_{1} e^{b t}, \quad b, B_{1}>0 .
$$

Substituting in (1.1), we have

$$
\left|x^{\prime}(t)\right| \leqq k+B_{1} e^{b t} \int_{0}^{1}[a(\tau)+c] d \tau+[a(1)+c] \int_{0}^{t} B_{1} e^{b \tau} d \tau \leqq B_{2} e^{b t} .
$$

Taking Laplace transforms in (1.1), we obtain $X(s) p(s)=x_{0}+(k / s), \operatorname{Re} s>b$, with $p(s)=c / s+A(s)+s$, as in Lemma 5. By Lemma $5(\mathrm{i})$,

$$
X(s)=\left(x_{0}+k / s\right) / p(s), \quad \operatorname{Re} s>b,
$$

and (3.1) defines $X(s)$ as a function holomorphic in $\{\operatorname{Re} s>0\}$ and continuous in $S$ (for H3) or in $S-\{ \pm i \omega\}$ (for H4). Also note that by (2.5) we have $[X(s)]^{*}=X\left(s^{*}\right)$; and by Lemma 3(ii), $X(\sigma+i \tau) \rightarrow 0$ as $|\tau| \rightarrow \infty$, uniformly in $0 \leqq \sigma<\infty$.

(i) We set $x_{0}=1, k=0, X=U$ in (3.1). Then

$$
U(s)=\frac{1}{s}-\frac{(c / s)+A(s)}{c+s A(s)+s^{2}}, \quad s=\sigma+i \tau \in S .
$$

For any $\sigma \geqq 0$ and sufficiently large $R>0$, the second term is in $L_{1}\{(-\infty,-R)$ $\cup(R, \infty)\}$ as a function of $\tau$, by Lemma 3(ii); and integration by parts shows that for any $T>0$

$$
\left[\int_{-\infty}^{-R}+\int_{R}^{\infty}\right] e^{i \tau t}(\sigma+i \tau)^{-1} d \tau
$$

converges uniformly for $t \geqq T$. Then the exponential bound on $u(t)$ and $u(t) \in C^{\prime}$ justify the inversion formula

$$
2 \pi u(t)=e^{\sigma t} \int_{-\infty}^{\infty} e^{i \tau t} U(\sigma+i \tau) d \tau, \quad \sigma>b, t>0 .
$$

If $c+a(t) \in L_{1}(0, \infty), A(s)$ has limit $A(0)=\int_{0}^{\infty} a(t) d t$ at $s=0$, so $U(s)$ is continuous with $U(0)=1 / A(0)$. If $c+a(t) \notin L_{1}(0, \infty)$, Corollaries 3.1 (if $c>0$ ) and 3.2 (if $c=0$ ) show that $U(s) \rightarrow 0$ as $s \rightarrow 0, s \in S$, and again $U(s)$ is continuous at $s=0$. 
Thus if (H3) holds, Cauchy's theorem and the fact that $U(\sigma+i \tau) \rightarrow 0$ as $|\tau|$ $\rightarrow \infty$ uniformly in $\sigma$ yield

$$
2 \pi u(t)=\int_{-\infty}^{\infty} e^{i \tau t} U(i \tau) d \tau
$$

The Riemann-Lebesgue theorem for finite intervals and the uniform convergence of the integral in (3.3) yield $u(t) \rightarrow 0$ as $t \rightarrow \infty$.

On the other hand, if (H4) holds, we set

$$
U_{1}(s)=U(s)-2 s / \gamma\left(s^{2}+\omega^{2}\right) .
$$

Since $2 s / \gamma\left(s^{2}+\omega^{2}\right)$ is the Laplace transform of $2 \gamma^{-1} \cos \omega t$, (3.2) holds with $u_{1}$ and $U_{1}$ in place of $u$ and $U$. Using Cauchy's theorem as before, we have for $0<\rho<\omega$,

$$
\begin{aligned}
2 \pi u_{1}(t)= & {\left[\int_{-\infty}^{-\omega-\rho}+\int_{-\omega+\rho}^{\omega-\rho}+\int_{\omega+\rho}^{\infty}\right] e^{i \tau t} U_{1}(i \tau) d \tau } \\
& +\frac{1}{i}\left[\int_{C_{\rho}^{-}}+\int_{C_{\rho}^{+}}\right] e^{s t} U_{1}(s) d s, \quad t>0,
\end{aligned}
$$

where $C_{\rho}^{ \pm}$is the semicircle $\left\{ \pm i \omega+\rho e^{i \theta},-\pi / 2 \leqq \theta \leqq \pi / 2\right\}$. Since $U_{1}\left(s^{*}\right)=\left[U_{1}(s)\right]^{*}$, this may also be written

$$
\pi u_{1}(t)=\left[\int_{0}^{\omega-\rho}+\int_{\omega+\rho}^{\infty}\right] \operatorname{Re}\left\{e^{i \tau t} U_{1}(i \tau)\right\} d \tau+\frac{1}{i} \int_{C_{\rho}^{+}} \operatorname{Re}\left\{e^{s t} U_{1}(s)\right\} d s, \quad t>0
$$

Note that

$$
U_{1}(s)=\frac{1}{p(s)}-\frac{1}{\gamma(s-i \omega)}+O(1) \quad(s \rightarrow i \omega, s \in S) .
$$

Writing $p(s)=\gamma(s-i \omega)+[p(s)-\gamma(s-i \omega)]$, we find that

$$
U_{1}(s)=\frac{p(s)-\gamma(s-i \omega)}{\gamma(s-i \omega)^{2}}\left[\frac{-1}{\gamma+(p(s)-\gamma(s-i \omega)) /(s-i \omega)}\right]+O(1) \quad(s \rightarrow i \omega, s \in S) .
$$

Thus, for $s \in C_{\rho}^{+},(2.13)$ yields $e^{s t} U_{1}(s)=o\left(\rho^{-1}\right),(\rho \rightarrow 0)$. Since $\left|C_{\rho}^{+}\right|=\pi \rho$, we may let $\rho \rightarrow 0$ in (3.5) and obtain for $0<\eta<\omega$

$$
\pi u_{1}(t)=\left[\int_{0}^{\omega-\eta}+\int_{\omega+\eta}^{\infty}\right] \operatorname{Re}\left\{e^{i \tau t} U_{1}(i \tau)\right\} d \tau+\lim _{\varepsilon \rightarrow 0}\left[\int_{\omega-\eta}^{\omega-\varepsilon}+\int_{\omega+\varepsilon}^{\omega+\eta}\right] \operatorname{Re}\left\{e^{i \tau t} U_{1}(i \tau)\right\} d \tau .
$$

Treating the first term as for (H3) (3.4 and integration by parts show that $\int_{\omega+\eta}^{\infty}$ converges uniformly), we have

$$
\begin{array}{r}
\pi u_{1}(t)=\lim _{\varepsilon \rightarrow 0}\left[\int_{\omega-\eta}^{\omega-\varepsilon}+\int_{\omega+\varepsilon}^{\omega+\eta}\right]\left\{\left[\operatorname{Re} U_{1}(i \tau)\right] \cos \tau t-\left[\operatorname{Im} U_{1}(i \tau)\right] \sin \tau t\right\} d \tau+o(1), \\
(t \rightarrow \infty), 0<\eta<\omega .
\end{array}
$$

For real $\lambda$ define

$$
S(\lambda)=\sum_{k=1}^{\infty} \frac{\delta_{k}}{k t_{0}}\left(\sin k t_{0} \lambda-k t_{0} \lambda\right), \quad C(\lambda)=\sum_{k=1}^{\infty} \frac{\delta_{k}}{k t_{0}}\left(\cos k t_{0} \lambda-1\right) .
$$


Note that

$$
C(\lambda)=C(-\lambda) \leqq 0, \quad \lambda S(\lambda)=-\lambda S(-\lambda) \leqq 0,
$$

and $C(\lambda+j \omega)=C(\lambda)$, for any integer $j$. By (2.14),

$$
p(i \tau)-i \gamma(\tau-\omega)=[-C(\tau)+i S(\tau-\omega)] / \tau^{2}+O\left(|\tau-\omega|^{2}\right) \quad(\tau \rightarrow \omega) .
$$

(2.15) and the argument following it show that

$$
|C(\lambda)|+|S(\lambda)|=o(\lambda), \quad \lambda \rightarrow 0 .
$$

Using these facts in (3.6) one computes

$$
\operatorname{Re} U_{1}(i \tau)=\frac{-C(\tau)}{(\tau-\omega)^{2} \tau^{2}\left[\gamma^{2}+o(1)\right]}+O(1) \quad(\tau \rightarrow \omega),
$$

and

$$
\operatorname{Im} U_{1}(i \tau)=R(\tau-\omega)+o\left(\frac{C(\tau)}{(\tau-\omega)^{2}}\right)+O(1) \quad(\tau \rightarrow \omega)
$$

where

$$
R(\lambda)=\frac{S(\lambda)\left[\gamma+S(\lambda) / \omega^{2} \lambda\right]}{\gamma \lambda^{2} \omega^{2}\left[\left(\gamma+S(\lambda) / \lambda \omega^{2}\right)^{2}+C^{2}(\lambda) / \lambda^{2} \omega^{4}\right]}
$$

Now let $t=t^{*}=2 \pi / \omega$ in (3.7). Since $\sin \tau t^{*}=\sin (\tau-\omega) t^{*}=O(\tau-\omega),(\tau \rightarrow \omega)$, we see from (3.9) and (3.11) that with $t=t^{*}$ the second term in the integrand in (3.7) is bounded on $(\omega-\eta, \omega+\eta)$. It follows from (3.7) and (3.10) that

$$
\lim _{\varepsilon \rightarrow 0}\left[\int_{\omega-\eta}^{\omega-\varepsilon}+\int_{\omega+\varepsilon}^{\omega+\eta}\right] \frac{C(\tau) \cos \tau t^{*} d \tau}{(\tau-\omega)^{2} \tau^{2}\left[\gamma^{2}+o(1)\right]}
$$

exists and is finite. But by the choice of $t^{*}$, the integrand in (3.12) is $\leqq$ $C(\tau) / 2(\tau-\omega)^{2} \gamma^{2} \omega^{2}$ for $|\tau-\omega|$ sufficiently small. Since $C(\tau) \leqq 0$ we conclude

$$
C(\tau) /(\tau-\omega)^{2} \in L_{1}(\omega-\eta, \omega+\eta) .
$$

In view of (3.10), (3.11), and (3.13), an application of the Riemann-Lebesgue theorem to (3.7) yields

$$
-\pi u_{1}(t)=\lim _{\varepsilon \rightarrow 0}\left[\int_{\omega-\eta}^{\omega-\varepsilon}+\int_{\omega+\varepsilon}^{\omega+\eta}\right] R(\tau-\omega) \sin \tau t d \tau+o(1), \quad t \rightarrow \infty .
$$

Note that $R(-\lambda)=-R(\lambda)$. The change of variables $\lambda=\tau-\omega$ in (3.14) shows that to complete the proof of (i) we need only show that

$$
r(t) \equiv \lim _{\varepsilon \rightarrow 0} \int_{\varepsilon}^{\eta}[\sin (\omega+\lambda) t-\sin (\omega-\lambda) t] R(\lambda) d \lambda=o(1) \quad(t \rightarrow \infty) .
$$

A trigonometric identity and $|(\sin \lambda t) / \lambda| \leqq t$ permit us to write

$$
r(t)=2 \cos (\omega t) \int_{0}^{\eta} R(\lambda) \sin \lambda t d \lambda .
$$


To prove (3.15), note first that

$$
\left|S^{\prime}(\lambda)\right|=\left|\sum_{k=1}^{\infty} \delta_{k}\left(\cos k t_{0} \lambda-1\right)\right| \leqq 2 \delta .
$$

Similarly, $\left|C^{\prime}(\lambda)\right| \leqq \delta$. Straightforward computations and estimates then show that $\left|R^{\prime}(\lambda)\right| \leqq M / \lambda^{2}$ for some constant $M<\infty, 0<\lambda<\eta$. Also note that $|\lambda R(\lambda)| \leqq$ $K_{1}|S(\lambda) / \lambda| \leqq K_{2}, K_{1}, K_{2}<\infty$.

Now let $\varepsilon>0$ be given; pick $\mu>0$ so that $\left(M+K_{2}\right)<\mu \varepsilon / 3$, and pick $T>0$ so that $\left(M+K_{2}\right) / \eta T \leqq \varepsilon / 3$ and $\left|K_{1} S(\lambda) / \lambda\right| \leqq \varepsilon / 3 \mu$ for $0<\lambda<\mu / T$. Then for $t \geqq T$, integration by parts yields

while

$$
\left|\int_{\mu / t}^{\eta} R(\lambda) \sin \lambda t d \lambda\right| \leqq\left(M+K_{2}\right)\left(\frac{1}{\eta T}+\frac{1}{\mu}\right) \leqq 2 \varepsilon / 3,
$$

$$
\left|\int_{0}^{\mu / t} R(\lambda) \sin \lambda t d \lambda\right| \leqq\left|\int_{0}^{\mu / t}[\lambda R(\lambda) \sin \lambda t] / \lambda d \lambda\right| \leqq \varepsilon / 3 .
$$

These estimates, together with (3.16), prove (3.15) and complete the proof of (i).

(ii) We set $x_{0}=0, k=1, X=W$ in (3.1). Note that $W(\sigma+i \tau)=O\left(\tau^{-2}\right),|\tau| \rightarrow \infty$, so $W(\sigma+i \tau) \in L_{1}\{(-\infty, R) \cup(R, \infty)\}$ for $R$ sufficiently large and $0 \leqq \sigma<\infty$.

If (H3) holds, we use Cauchy's theorem as in (i) to obtain, for each $\varepsilon>0$ sufficiently small,

$$
2 \pi w(t)=\left[\int_{-\infty}^{-\varepsilon}+\int_{\varepsilon}^{\infty}\right] e^{i \tau t} W(i \tau) d \tau+\frac{1}{i} \int_{D_{\epsilon}} e^{s t} W(s) d s,
$$

where $D_{\varepsilon}$ is the semicircle $\left\{\varepsilon e^{i \theta},-\pi / 2 \leqq \theta \leqq \pi / 2\right\}$. Corollaries 3.1 and 3.2 show that $W\left(\varepsilon e^{i \theta}\right)=o(1 / \varepsilon),(\varepsilon \rightarrow 0)$, uniformly in $-\pi / 2 \leqq \theta \leqq \pi / 2$. Since $\left|D_{\varepsilon}\right|=\varepsilon \pi$, we may let $\varepsilon \rightarrow 0$ in (3.17) and obtain

$$
2 \pi w(t)=\lim _{\varepsilon \rightarrow 0}\left[\int_{-\infty}^{-\varepsilon}+\int_{\varepsilon}^{\infty}\right] e^{i \tau t} W(i \tau) d \tau, \quad t>0
$$

Now the symmetry of $W(i \tau)$ in $\tau$ and the Riemann-Lebesgue theorem imply that for $\Delta>0$,

$$
\pi w(t)=\lim _{\varepsilon \rightarrow 0} \int_{\varepsilon}^{\Delta} \operatorname{Re}\left\{e^{i \tau t} W(i \tau)\right\} d \tau+o(1) \quad(t \rightarrow \infty)
$$

Next we derive a formula similar to (3.18) for $w_{1}(t)$ in the (H4) case. Define

$$
W_{1}(s)=W(s)-2 / \gamma\left(s^{2}+\omega^{2}\right) .
$$

Note that $2 / \gamma\left(s^{2}+\omega^{2}\right)$ is the Laplace transform of $2(\gamma \omega)^{-1} \sin \omega t$. Note also that $W_{1}(s)=U_{1}(s) / s=\left(U_{1}(s) / \omega\right)+O(1),(s \rightarrow \omega, s \in S)$. Continuing as with $U_{1}(s)$ in (i) and using $W_{1}(i \tau) \in L_{1}(\omega+\eta, \infty)$, we obtain

$$
\begin{aligned}
\pi w_{1}(t)= & \lim _{\varepsilon \rightarrow 0} \int_{\varepsilon}^{\omega-\eta} \operatorname{Re}\left\{e^{i \tau t} W_{1}(i \tau)\right\} d \tau \\
& +\omega^{-1} \lim _{\rho \rightarrow 0}\left[\int_{\omega-\eta}^{\omega-\rho}+\int_{\omega+\rho}^{\omega+\eta}\right] \operatorname{Re}\left\{e^{i \tau t} U_{1}(i \tau)\right\} d \tau+o(1), \\
t \rightarrow \infty, 0<\eta<\omega . &
\end{aligned}
$$


Since $W_{1}(s)-W(s)$ is bounded on $(0, \omega-\eta)$, we may replace $W_{1}$ by $W$ in the first term above. By (3.7) and conclusion (i) of the theorem, the second term is $o(1),(t \rightarrow \infty)$. Thus for $0<\Delta<\omega$,

$$
\pi w_{1}(t)=\lim _{\varepsilon \rightarrow 0} \int_{\varepsilon}^{\Delta} \operatorname{Re}\left\{e^{i \tau t} W_{1}(i \tau)\right\} d \tau+o(1) \quad(t \rightarrow \infty) .
$$

Now set $\Delta=1$ when (H3) holds, $\Delta=\omega / 2$ when (H4) holds. By (3.18) and (3.19) we can complete the proof by showing that

$$
\lim _{t \rightarrow \infty}\left(\lim _{\varepsilon \rightarrow \infty} \int_{\varepsilon}^{\Delta} \operatorname{Re}\left\{e^{i \tau t} W(i \tau)\right\} d \tau\right)=0 .
$$

If $c>0, W(i \tau)$ is continuous on $[0, \Delta]$, by Corollary 3.1 , so that $(3.20)$ holds. It remains to prove (3.20) with $c=0$.

For $0<\tau \leqq \Delta$, define
(a) $\phi_{1}(i \tau)=\int_{0}^{\pi / 2 \tau} a(t) \cos \tau t d t$
(b) $\phi_{2}(i \tau)=\lim _{T \rightarrow \infty} \int_{\pi / 2 \tau}^{T} a(t) \cos \tau t d t$
(c) $\phi(i \tau)=\phi_{1}(i \tau)+\phi_{2}(i \tau)$
(d) $\psi(i \tau)=\lim _{T \rightarrow \infty} \int_{0}^{T} a(t) \sin \tau t d t$.

As in the proof of Lemma 3,

$$
\operatorname{Re} A(i \tau)=\phi(i \tau) \text { and } \operatorname{Im} A(i \tau)=-\psi(i \tau) .
$$

Lemma 3 gives some useful facts about these functions. In addition, since $0 \leqq a(t) \downarrow, \phi_{2}(i \tau) \leqq 0$, so that (2.4) implies

$$
\psi(i \tau) \geqq\left|\phi_{2}(i \tau)\right|, \quad 0<\tau \leqq \Delta .
$$

Also, (2.1), (3.22), and $0 \leqq a(t) \downarrow$ yield

$$
\begin{aligned}
0 \leqq \psi(i \tau) & \leqq \int_{0}^{\pi / \tau} a(t) \sin \tau t d t \\
& \leqq 4 \int_{0}^{\pi / 4 \tau} a(t) \cos \tau t d t \\
& \leqq 4 \int_{0}^{\pi / 2 \tau} a(t) \cos \tau t d t
\end{aligned}
$$

i.e.,

$$
0 \leqq \psi(i \tau) \leqq 4 \phi_{1}(i \tau), \quad 0<\tau \leqq \Delta .
$$

The choice of $\lrcorner$ insures that $W(i \tau)$ is continuous on $(0, \Delta]$. Using (3.22) and our assumption $c=0$, we compute

$$
W(i \tau)=\frac{(\psi(i \tau)-\tau)}{\tau|A(i \tau)+i \tau|^{2}}-i \frac{\phi(i \tau)}{\tau|A(i \tau)+i \tau|^{2}} .
$$


Defining $y(t)=\lim _{\varepsilon \rightarrow 0} \int_{\varepsilon}^{\Delta} \operatorname{Re}\left\{e^{i \tau t} W(i \tau)\right\} d \tau$, we have

$$
y(t)=\lim _{\varepsilon \rightarrow 0}\left[\int_{\varepsilon}^{\Delta} \cos \tau t \frac{\psi(i \tau) d \tau}{\tau|A(i \tau)+i \tau|^{2}}-\int_{\varepsilon}^{\Delta} \frac{\cos \tau t d \tau}{|A(i \tau)+i \tau|^{2}}+\int_{\varepsilon}^{\Delta} \frac{\sin \tau t}{\tau} \frac{\phi(i \tau) d \tau}{|A(i \tau)+i \tau|^{2}}\right] .
$$

By Corollary 3.2, the middle integrand is continuous on $[0, \Delta]$. Since $|\sin \tau t / \tau| \leqq t$ for $t>0$ and

$$
\frac{|\phi(i \tau)|}{|A(i \tau)+i \tau|}=\frac{|\phi(i \tau)|}{|\phi(i \tau)+i(\tau-\psi(i \tau))|} \leqq 1
$$

Corollary (3.2) also shows that the third integrand is continuous in $[0, \Delta]$ for each $t$. Therefore

$$
\begin{aligned}
y(t)= & \lim _{\varepsilon \rightarrow 0} \int_{\varepsilon}^{\Delta} \cos \tau t \frac{\psi(i \tau) d \tau}{\tau|A(i \tau)+i \tau|^{2}}-\int_{0}^{\Delta} \frac{\cos \tau t d \tau}{|A(i \tau)+i \tau|^{2}} \\
& +\int_{0}^{\Delta} \frac{\sin \tau t}{\tau} \frac{\phi(i \tau) d \tau}{|A(i \tau)+i \tau|^{2}}, \quad t>0 .
\end{aligned}
$$

But for $0<\tau<\pi / 3 t$ we have $\cos \tau t>1 / 2$ and $\psi(i \tau) \geqq 0$; hence the existence of the limit in (3.25) shows that

$$
\psi(i \tau) / \tau|A(i \tau)+i \tau|^{2} \in L_{1}(0, \Delta)
$$

Applying the Riemann-Lebesgue theorem to the first two integrals in (3.25), we obtain

$$
y(t)=\int_{0}^{\Delta} \frac{\sin \tau t}{\tau} \frac{\phi(i \tau) d \tau}{|A(i \tau)+i \tau|^{2}}+o(1) \quad(t \rightarrow \infty) .
$$

Another consequence of (3.26), together with (3.23), is

$$
\phi_{2}(i \tau) / \tau|A(i \tau)+i \tau|^{2} \in L_{1}(0, \Delta) .
$$

We rewrite (3.27) as

$$
\begin{aligned}
y(t) & =\int_{0}^{\Delta} \frac{\sin \tau t}{\tau} \frac{d \tau}{\phi_{1}(i \tau)}+\int_{0}^{\Delta} \frac{\sin \tau t}{\tau} \frac{\phi_{1}(i \tau) \phi(i \tau)-\phi^{2}(i \tau)-(\psi(i \tau)-\tau)^{2}}{\phi_{1}(i \tau)|A(i \tau)+i \tau|^{2}} d \tau+o(1) \\
& =y_{1}(t)+y_{2}(t)+o(1) \quad(t \rightarrow \infty) .
\end{aligned}
$$

Then

$$
y_{2}(t)=-\int_{0}^{\Delta} \sin \tau t\left[\frac{\phi_{1}(i \tau)+\phi_{2}(i \tau)}{\phi_{1}(i \tau)} \phi_{2}(i \tau)+\frac{\psi(i \tau)-\tau}{\phi_{1}(i \tau)}(\psi(i \tau)-\tau)\right] \frac{d \tau}{\tau|A(i \tau)+i \tau|^{2}}
$$

Now, (3.23) and (3.24) show that $\left|\left(\phi_{1}(i \tau)+\phi_{2}(i \tau)\right) / \phi_{1}(i \tau)\right|$ and $\left|\psi(i \tau) / \phi_{1}(i \tau)\right|$ are bounded on $(0, \Delta)$. Since $a(t) \notin L_{1}(0, \infty)$, (3.21a) gives

$$
\phi_{1}(i \tau) \geqq \frac{1}{2} \int_{0}^{\pi / 3 \tau} a(t) d t \rightarrow \infty, \quad \text { as } \tau \rightarrow 0,
$$

so that $\left|\tau / \phi_{1}(i \tau)\right|$ is also bounded. Then by (3.26), (3.28), and Corollary 3.2, the 
coefficient of $\sin \tau t$ in (3.30) is in $L_{1}(0, \Delta)$. Using the Riemann-Lebesgue theorem once more, we have

$$
\lim _{t \rightarrow \infty} y_{2}(t)=0 \text {. }
$$

Finally, to treat $y_{1}(t)$, we note that for $\tau>0$

$$
\frac{d}{d \tau} \phi_{1}(i \tau)=-\int_{0}^{\pi / 2 \tau} t a(t) \sin \tau t d t \leqq 0 .
$$

Thus by (3.31), $1 / \phi_{1}(i \tau) \downarrow 0$ as $\tau \downarrow 0$; in particular, $1 / \phi_{1}(i \tau)$ is of bounded variation on $[0, \Delta]$, and a familiar theorem concerning the kernel $(\sin \tau t) / \tau[8, \mathrm{p} .64]$ yields $y_{1}(t) \rightarrow 0$ as $t \rightarrow \infty$. Combining this with (3.29) and (3.32), we have (3.20), and (ii) is proved.

(iii) A proof similar to that for (ii) can be obtained by considering $W_{2}(s)$ $\equiv W(s)-1 / A(0) s$. We obtain (iii) from (i) as done in the proof of Theorem 2(i) of [5].

By the definition of $w(t)$, we have $u(t)=w^{\prime}(t)$, where the asymptotic behavior of $u(t)$ is given by conclusion (i). (1.1) for $w$ becomes

$$
u(t)-1=-\int_{0}^{t} a(t-\tau) w(\tau) d \tau
$$

since $c+a(t) \in L_{1}(0, \infty)$ implies $c=0$.

When (H3) holds, Levin's proof of Theorem 2(i) in [5] applies word for word to give $w(t) \rightarrow\left(\int_{0}^{\infty} a(t) d t\right)^{-1}$ as $t \rightarrow \infty$.

If (H4) holds, we use $A(i \omega)=-i \omega$ (from Lemma 5) and $a(t) \in L_{1}(0, \infty)$ to compute

$$
\begin{aligned}
-\int_{0}^{t} a(t-\tau) \frac{2 \sin \omega \tau d \tau}{3 \omega} & =\frac{-2}{3 \omega} \int_{0}^{t} a(\tau) \sin [\omega(t-\tau)] d \tau \\
& =\operatorname{Im}\left\{\frac{-2 e^{i \omega t}}{3 \omega}\left[A(i \omega)-\int_{t}^{\infty} a(\tau) e^{-i \omega \tau} d \tau\right]\right\} \\
& =\frac{2 \cos \omega t}{3}+o(1) \quad(t \rightarrow \infty) .
\end{aligned}
$$

Then by (3.33) and conclusion (i),

$$
\int_{0}^{t} a(t-\tau) w_{1}(\tau) d \tau=1+o(1) \quad(t \rightarrow \infty)
$$

and $w_{1}^{\prime}(t) \rightarrow 0$ as $t \rightarrow \infty$. The proof of (iii) can now be completed by the method of Theorem 2(i) of [5], which gives $w_{1}(t) \rightarrow\left(\int_{0}^{\infty} a(t) d t\right)^{-1}$ as $t \rightarrow \infty$. This completes the proof of the theorem.

\section{REFERENCES}

1. R. Bellman and K. Cooke, Differential-difference equations, Academic Press, New York, 1963. 
2. G. Doetsch, Theorie und Anwendung der Laplace-Transformation, Springer-Verlag, Berlin, 1937.

3. A. Friedman, On integral equations of Volterra type, J. Analyse Math. 11 (1963), 381-413.

4. A. Halanay, On the asymptotic behavior of the solutions of an integro-differential equation, J. Math. Anal. Appl. 10 (1965), 319-324.

5. J. J. Levin, The asymptotic behavior of the solution of a Volterra equation, Proc. Amer. Math. Soc. 14 (1963), 534-541.

6. J. J. Levin and J. A. Nohel, On a system of integrodifferential equations occurring in reactor dynamics, J. Math. Mech. 9 (1960), 347-368.

7. - Perturbations of a nonlinear Volterra equation, Michigan Math. J. 12 (1965), 431-447.

8. D. V. Widder, The Laplace transform, Princeton Univ. Press, Princeton, N. J., 1946.

UNIVERSITY OF CALIForNiA,

los Angeles, California 